\title{
Supergiant Fast X-ray Transients - A short review
}

\section{Lara Sidoli*}

INAF/IASF Milano Italy

E-mail: sidelidiasf-milane.inaf.it

I present a brief up-to-date review of the current understanding of Supergiant Fast X-ray Transients, with an emphasis on the observational point of view. After more than a decade since their discovery, a remarkable progress has been made in getting the picture of their phenomenology at X-ray energies. However, a similar in-depth investigation of the properties of the supergiant companions is needed, but has started more recently. A multifrequency approach is the key to fully understand the physical mechanism driving the SFXT behaviour, still under debate.

XII Multifrequency Behaviour of High Energy Cosmic Sources Workshop

12-17 June 2017

Palermo, Italy

${ }^{*}$ Speaker. 


\section{The strange case of SFXTs}

In this short review paper I will concentrate on the most recent achievements about Supergiant Fast X-ray Transients (SFXTs), emphasizing an observational standpoint. The X-ray window will be looked at in particular, but with an eye also to observations at other frequencies, especially for what concerns the future directions. I will mainly focus on advances on the topic obtained thanks to systematic investigations of SFXT class as a whole, while I refer the reader to [四] for a summary of the properties of the single sources. For a unique, comprehensive and detailed review of the current understanding of the interplay between the outflowing wind from massive stars and accretion processes in wind-fed supergiant HMXBs, I refer the reader to []].

SFXTs ([目], [四]) are a sub-class of High Mass X-ray Binaries (HMXBs) where a compact object (typically a neutron star) accretes a fraction of the clumpy wind from the blue supergiant companion, triggering rare and short (less than a few days) outbursts, made of a number of bright $\mathrm{X}$-ray flares (each flare with a duration of $\sim$ thousands seconds). Most SFXTs were discovered by the INTEGRAL satellite (launched in 2002; [5]) as hard (above $17 \mathrm{keV}$ ) X-ray sources detected only during a short duration (a few hours) X-ray activity. Thanks to X-ray sky positions refined at arcsec level at soft X-rays (1-10 keV), they were quickly associated with $\mathrm{O}$ or B-type supergiants. The identification of these $\mathrm{X}$-ray transients with massive $\mathrm{X}$-ray binaries was somehow surprising, since the classical HMXBs with supergiant companion (SgHMXBs) known since the birth of Xray astronomy are persistent $\mathrm{X}$-ray emitters, with a limited range of intensity variability (around a factor of 10). Fig. W shows the comparison between long-term X-ray INTEGRAL light curve of an SFXT and Vela X-1, the prototype of persistent HMXBs.

Since their discovery, more than a decade ago, a huge observational effort has involved both

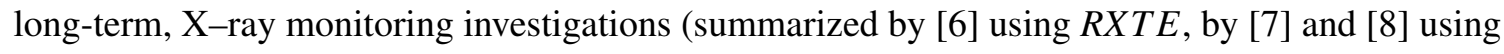
INTEGRAL, by [Q] and [0] using Swift) and deep, very sensitive, X-ray pointings (by Chandra, e.g. [Ш]], and XMM-Newton, starting from the pioneering observations discussed by [ए2], to the

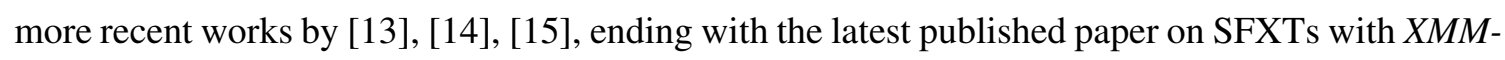
Newton [ए]]).

These X-ray observations led to the characterization of the SFXT properties, as follows:

1. low duty cycle $(\lesssim 5 \%)$ in bright $\mathrm{X}$-ray flares (at $\mathrm{L}_{X} \gtrsim 10^{36} \mathrm{erg} \mathrm{s}^{-1}$ )

2. high dynamic range (ratio between maximum and minimum $\mathrm{X}$-ray luminosity) $\gtrsim 100$

3. low time-averaged luminosity $\mathrm{L}_{X} \lesssim 10^{34} \mathrm{erg} \mathrm{s}^{-1}$

Within the above limits, a variety of behaviours is present among the members of the class. The duty cycle (percentage of time spent in bright X-ray activity) can reach very low values, as low as $0.1 \%$ (IGR J08408-4503, [四]). The dynamic range of their X-ray emission, between quiescence and flare peaks, can spand values between $\sim 100$ (in this case the source is named "intermediate SFXT"), and $10^{6}$, reached only in IGR 17544-2619, the prototypical SFXT where the X-ray luminosity variations are carried to their extremities, from $10^{32} \mathrm{erg} \mathrm{s}^{-1}$ ([ए]]], [ए]3]) to $10^{38} \mathrm{erg} \mathrm{s}^{-1}$ [미].

Although it is evident from long-term Swift/XRT monitoring observations of a sample of SFXTs that the time-averaged X-ray luminosity is $\mathrm{L}_{X} \lesssim 10^{34} \mathrm{erg} \mathrm{s}^{-1}$ ([ए8], [Q] ] and [ए]]), Swift/XRT 


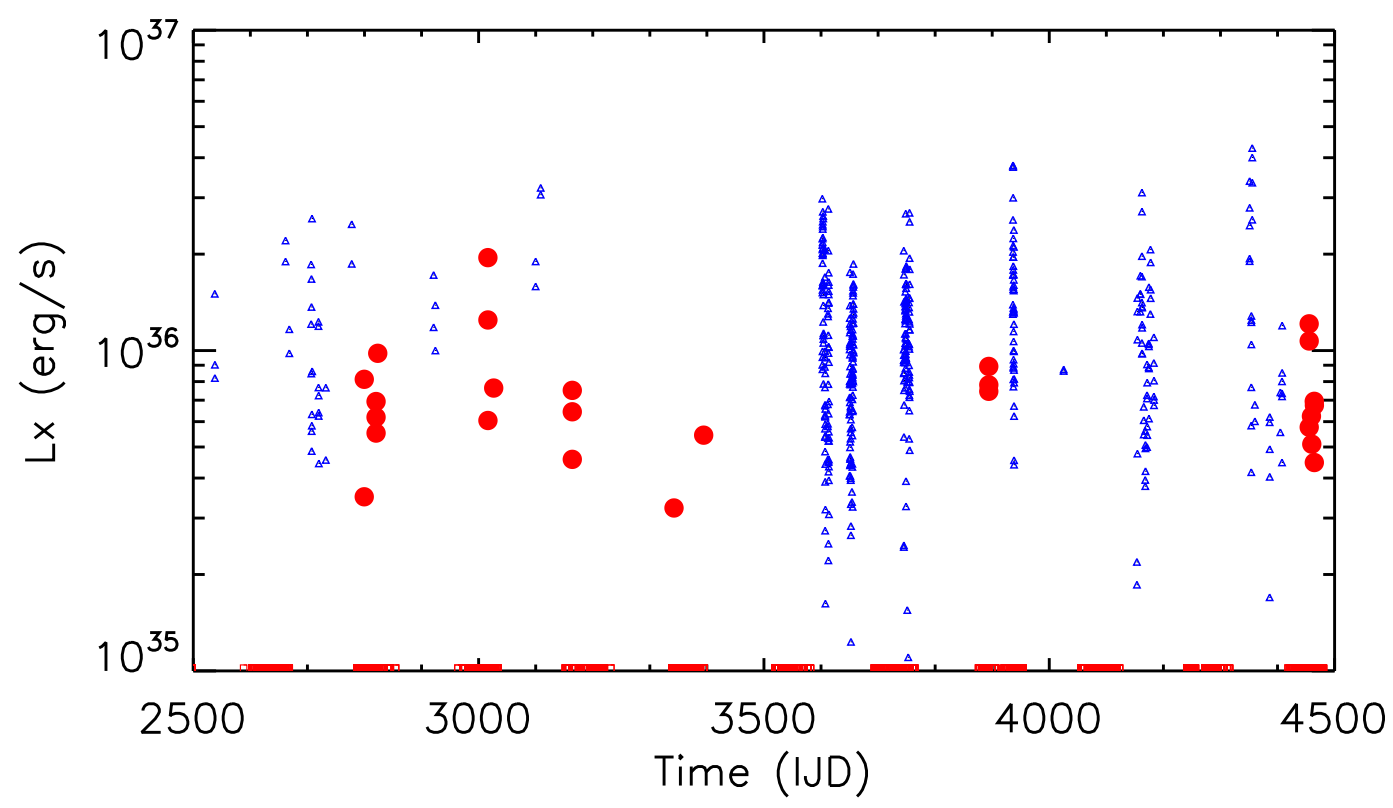

Figure 1: Comparison between INTEGRAL/IBIS light curves (bin time of $2 \mathrm{ks} ; 17-50 \mathrm{keV}$ ) of Vela X-1 (blue triangles) and an SFXT (IGR J17544-2619; each red solid cirle mark a flare). Red bars on the $\mathrm{x}$-axis indicate times when IGR J17544-2619 was undetected by INTEGRAL (its duty cycle is $\lesssim 1 \%$ ). On the other hand, Vela $\mathrm{X}-1$ is always detected. Time is in units of INTEGRAL JD (IJD=MJD-51544).

is not sensitive enough to reach very low fluxes and characterize the lowest luminosity states with short snapshots. The percentage of time spent below the Swift/XRT sensitivity threshold $\left(\sim 2 \times 10^{-12} \mathrm{erg} \mathrm{cm}^{-2} \mathrm{~s}^{-1}\right)$ ranges from $5 \%$ to $67 \%$, depending on the source [U]]. For instance, there are three SFXTs (IGR J08408-4503, IGR J16328-4726 and IGR J17544-2619) that remained undetected by Swift/XRT most of the time (67\%,61\% and 55\%, respectively), with a 2-10 keV luminosity below $2.6 \times 10^{33} \mathrm{erg} \mathrm{s}^{-1}$ (IGR J08408-4503), $2.5 \times 10^{34} \mathrm{erg} \mathrm{s}^{-1}$ (IGR J16328-4726) and $2.1 \times 10^{33} \mathrm{erg} \mathrm{s}^{-1}$ (IGR J17544-2619).

Very low quiescent luminosities of $\mathrm{L}_{X} \sim 10^{32} \mathrm{erg} \mathrm{s}^{-1}$ have been caught only in a few SFXTs thanks to XMM-Newton, Chandra, Suzaku pointings (e.g. []] [ [प]], [ए3]). Remarkably, the lowest X-ray emission state ever reported in an SFXT was observed in SAX J1818.6-1703 using XMMNewton, leading to a 3- $\sigma$ upper limit of $\mathrm{F}_{X}<1.1 \times 10^{-13} \mathrm{erg} \mathrm{cm}^{-2} \mathrm{~s}^{-1}$ (corrected for the absorption, $0.5-10 \mathrm{keV}$; [प⿴囗十)]). This implies an upper limit to its luminosity of $\mathrm{L}_{X}<6 \times 10^{31} \mathrm{erg} \mathrm{s}^{-1}$ (at $2.1 \mathrm{kpc}$ ). At present, it is unknown how much time each SFXT spends in quiescence at a luminosity level of $10^{32} \mathrm{erg} \mathrm{s}^{-1}$. It is also possible that some more active ("intermediate") SFXTs never reach it. A monitoring programme with XMM-Newton would be (unrealistically) needed, for this purpose.

The ample range of observed behaviours has posed an issue about the possible in-homogeneity of the SFXT class and about the membership of some specific sources (e.g. the case of IGR J164184532 or IGR J16465-4507, e.g. [U]], [四] opposite to the classification reported by [प]). IGR J112155259 is also peculiar, since it is the only SFXT where X-ray outbursts are strictly periodic [20], recurring every $\sim 165$ days ([2]], [[2]]), believed to be the orbital period of the system. In general, 
also X-ray flares from other SFXTs appear more concentrated around the periastron passage (e.g. [D2] ]), but nevertheless they can occur also at other orbital phases ([24], [焑]) and remarkably, in all sources but IGR J11215-5259, not every periastron passage triggers luminous flares. For example, after the recent determination of the orbital geometry in IGR J08408-4503 ([23] ), it is now evident that the long X-ray observation performed with Suzaku by [25] covered a large orbital phase interval just around periastron, and no bright flares were caught. However, it is important to remark that in a few SFXTs the orbital period has been discovered thanks to a periodic modulation in their long-term X-ray light curve (sometimes still present after removing bright flares).

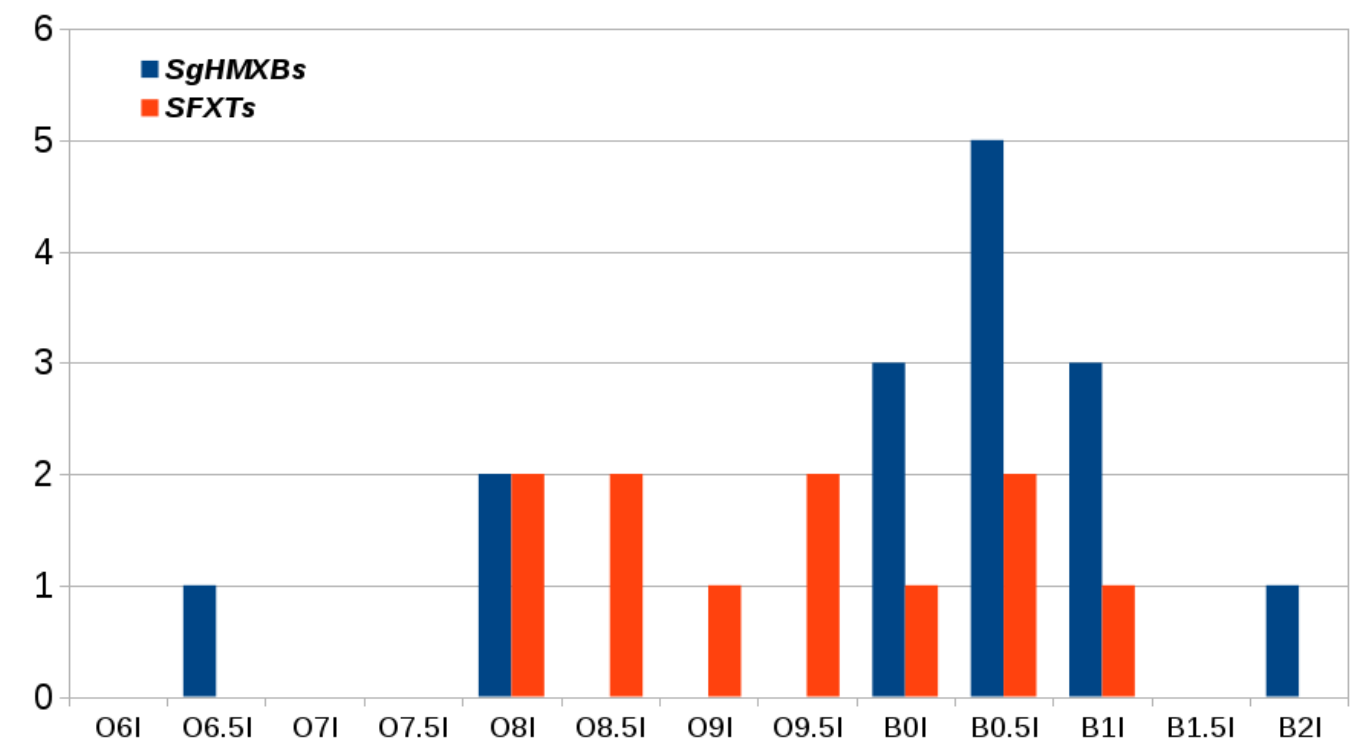

Figure 2: Spectral type of the supergiant companions in classical SgHMXBs (in blue, taken from the list reported by [[]]) and in SFXTs (in orange, taken from Table $\mathbb{\text { I) }}$.

In Table $\mathbb{W}$ an updated list of SFXTs is reported with some interesting quantities (spectral type of the supergiant companion, source distance, orbital geometry, spin period and super-orbital periodicities). Some of these properties are apparently very similar to those displayed by some persistent SgHMXBs: the spectral types of the massive companion overlap in the two kinds of HMXBs (Fig. Z, although O-type donors might appear more popular among SFXTs than in classical SgHMXBs); SgHMXBs have orbital periods overplapping with those shown by SFXTs, as well as pulsar spin periods (Fig. B]), although SFXTs orbital periodicities span a wider range of values.

X-ray pulsars appear to be more elusive among SFXTs, despite extensive searches; this might be due either to the large X-ray variability during flaring activity (hampering the detection of pulsations) or to the fact that some spin periods might be very long. The spin period in IGR J17544-2619 is controversial (marked by a question mark in Fig. (B)), since it has been found with RXTE/PCA data, and might be due to an another transient source within the field of view [26]. Alternatively, the lack of pulsations in many SFXTs could indicate that the compact object is a black hole, but the similarity of their X-ray spectra with those displayed by accreting X-ray pulsars (flat power-law within $10 \mathrm{keV}$, with a cutoff around $10-30 \mathrm{keV}$ ) suggests to exclude this possibility. 


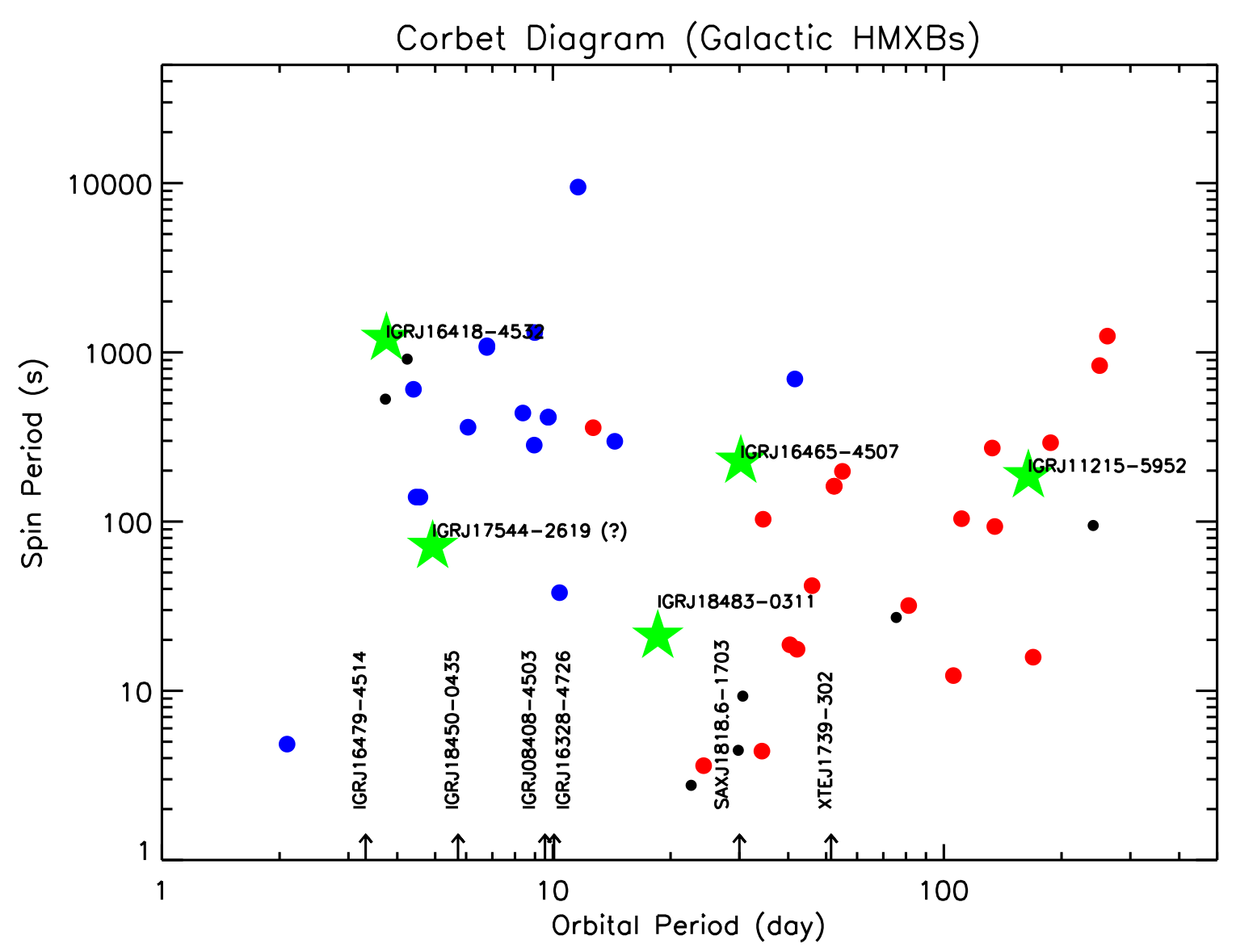

Figure 3: Spin period versus orbital period for Galactic High Mass X-ray Binaries. Blue dots indicate HMXBs hosting supergiant companions (excluding SFXTs, that are marked with green stars), while the red dots mark Be/XRBs. Arrows on the $\mathrm{x}$-axis mark the orbital periods of SFXTs with still unknown pulsar spin periods.

\section{Proposed explanations for the SFXT behaviour}

The whole core debate on SFXTs revolves around the physical mechanism producing their Xray phenomenology, at odds with what is observed in persistent SgHMXBs: their low percentage of time spent in bright $\mathrm{X}$-ray flares, their large $\mathrm{X}$-ray intensity variability between quiescence and flare peaks, their low time-averaged X-ray emission.

At first, it was suggested that the sporadic flares are produced by the direct (Bondi-Hoyle) accretion of supergiant wind clumps onto the neutron star ([प]]). But in this case, the estimated mass of the clumps can reach huge values $\left(10^{21}-10^{23} \mathrm{~g}\right)$, too large compared with what is known about hot stellar winds (clump masses $\lesssim 10^{18} \mathrm{~g}$; []]). It was demonstrated by [ [ $[\mathrm{U}]$ ] that the direct accretion from clumpy winds cannot account for SFXTs light curves, thus requiring a mechanism able to reduce accretion rate most of the time and to produce the rare and luminous X-ray flaring activity with the needed dynamic range.

Nowadays, the most debated explanations for the SFXT behaviour are the centrifugal (or magnetic) inhibition of accretion ("gating" mechanisms) at the neutron star magnetosphere ([2], [ㄱ] )

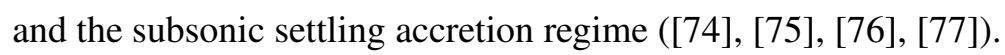


Table 1: Properties of SFXTs.

\begin{tabular}{|c|c|c|c|c|c|c|}
\hline Name & Companion & $\begin{array}{l}\text { Distance } \\
(\mathrm{kpc})\end{array}$ & $\begin{array}{l}\text { Orbital } \\
\text { Period (d) }\end{array}$ & ecc & $\begin{array}{l}\text { Spin } \\
\text { Period (s) }\end{array}$ & $\begin{array}{l}\text { Super-Orbital } \\
\text { Period (d) }\end{array}$ \\
\hline IGR J08408-4503 & $08.5 \mathrm{Ib}[2]$ & $2.7^{[28]}$ & $9.5436^{[23]}$ & $0.63^{[23]}$ & - & $285 \pm 10^{[23]}$ \\
\hline IGR J11215-5952 & B0.5Ia ${ }^{[29],[B], ~ B] ~}$ & $>7$ [B] & $164.6,[32,[2],[3]$ & $>0.8$ [3] & 187 [34, ㄷ] & - \\
\hline IGR J16328-4726 & O8Iafpe ${ }^{[B 5],[B],[B]}$ & $7.2[\mathrm{BB}]$ & $\left.10.068^{[\mathrm{Bg}}, \mathrm{40}\right]$ & - & - & - \\
\hline IGR J16418-4532 & $\mathrm{OB} \mathrm{Sg}$ & $\sim 13$ 四 & $3.753^{[-2]}$ & - & $1212^{\text {[4] }}$ & 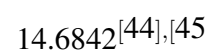 \\
\hline IGR J16465-4507 & O9.5Ia & $9.5^{[4]}$ & 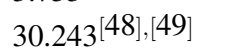 & - & 228 & - \\
\hline IGR J16479-4514 & O8.5I, O9.5Iab [1], [2] & $4.9,2.8$ 四, [2] & 3.3194 [53], [22] & - & - & $\left.11.880^{[44,}, 45\right]$ \\
\hline XTE J1739-302 & O8Iab(f) ${ }^{[?],[]]}$ & 2.7 西 & 51.47 [1] & - & - & - \\
\hline IGR J17544-2619 & $\mathrm{O} 9 \mathrm{Ib}[5]$ & 3.0 [ & $4.926^{[-6]}$ & $<0.4^{[6]}$ & $71.49^{[26]}$ & - \\
\hline IGR J17354-3255 & $\mathrm{OB} \mathrm{Sg}(?)$ & $8.5^{[-7]}$ & 8.4474 [18], [6] & - & - & - \\
\hline SAX J1818.6-1703 & $\sim \mathrm{BO}[$ [ & $2,2.1[6],[6]$ & $30.0[-6]$ & - & - & - \\
\hline IGR J18410-0535 & $\mathrm{B} 1 \mathrm{Ib}$ & $3.2^{49}$ & - & - & - & - \\
\hline IGR J18450-0435 & O9.5I & $3.6^{46]}$ & $5.7195^{[24]}$ & - & - & - \\
\hline IGR J18462-0223 & OB Sg(?) & $11(?)^{[6]}$ & $2.13(?)$ & - & - & - \\
\hline IGR J18483-0311 & $\mathrm{B} 0.5 \mathrm{Ia}[6]$ & $3^{367]}$ & $18.55^{[68]}$ & $\sim 0.4$ [6] & 21.0526 [प] & - \\
\hline
\end{tabular}

Gating mechanisms critically depend on the pulsar spin period and the neutron star magnetic field (see [[Z3] for a review), in presence of a variability in the density and velocity of the clumpy wind at the neutron star orbit. The centrifugal barrier can be at work in neutron stars with standard magnetic fields $\left(\mathrm{B} \sim 10^{12} \mathrm{G}\right)$ and pulsar spin periods of $\sim 10 \mathrm{~s}$, assuming blue supergiants winds with typical parameters (terminal velocity $\sim 1000 \mathrm{~km} \mathrm{~s}^{-1}$, mass-loss rate $\dot{M} \sim 10^{-6} M_{\odot} \mathrm{yr}^{-1}$ ). Of course, this also depends on the orbital geometry. In presence of longer spin periods, the neutron star magnetic field should be higher, for the centrifugal barrier to be closed. The magnetic barrier operates in slow pulsars (spin periods exceeding $1000 \mathrm{~s}$ ) with magnetar-like magnetic fields $\left(\mathrm{B} \sim 10^{14} \mathrm{G}\right)$. These mechanisms prevent accretion onto the neutron star most of the time, but the encounter with a dense clump along the orbit might temporarily allow the accretion, triggering an X-ray flare. Gating mechanisms require that SFXTs host slow spin periods (>1000 s) and very strong magnetic fields, to reproduce their light curves [प8], implying that SFXTs should host slower pulsars with much higher magnetic fields than in persistent SgHMXBs.

Neutron star magnetic fields (directly measured from the detection of cyclotron resonance scattering features in the X-ray spectrum) determined to-date in SFXTs are the following: a hint of a low magnetic field $\left(\mathrm{B} \sim 10^{11} \mathrm{G}\right)$ has been obtained in IGR J18483-0311 ([प्Q]), while a few $10^{12} \mathrm{G}$ has been measured in IGR J17544-2619 from the detection of a cyclotron line at $\sim 17 \mathrm{keV}$ in the NuSTAR spectrum [ $[\mathbb{8 0}]$. However the presence of this cyclotron line was not confirmed by []] during a second NuSTAR observation that caught the source at similar X-ray fluxes, possibly suggesting a variability of the cyclotron line. Note also that [ [ 8$]$ failed to detect any pulsar period from IGR J17544-2619. A search for a cyclotron line in the XMM-Newton plus NuSTAR X-ray spectrum of the SFXT pulsar IGR J11215-5952 [U5]] resulted in a hint of an absorption feature at 17 $\mathrm{keV}$ during a couple of flares. With an estimated line significance at $2.63 \sigma$, this line needs further confirmation (see Fig. $\$$ for the source light curve in outburst, and the time-averaged spectrum during the observation performed in February 2016). 

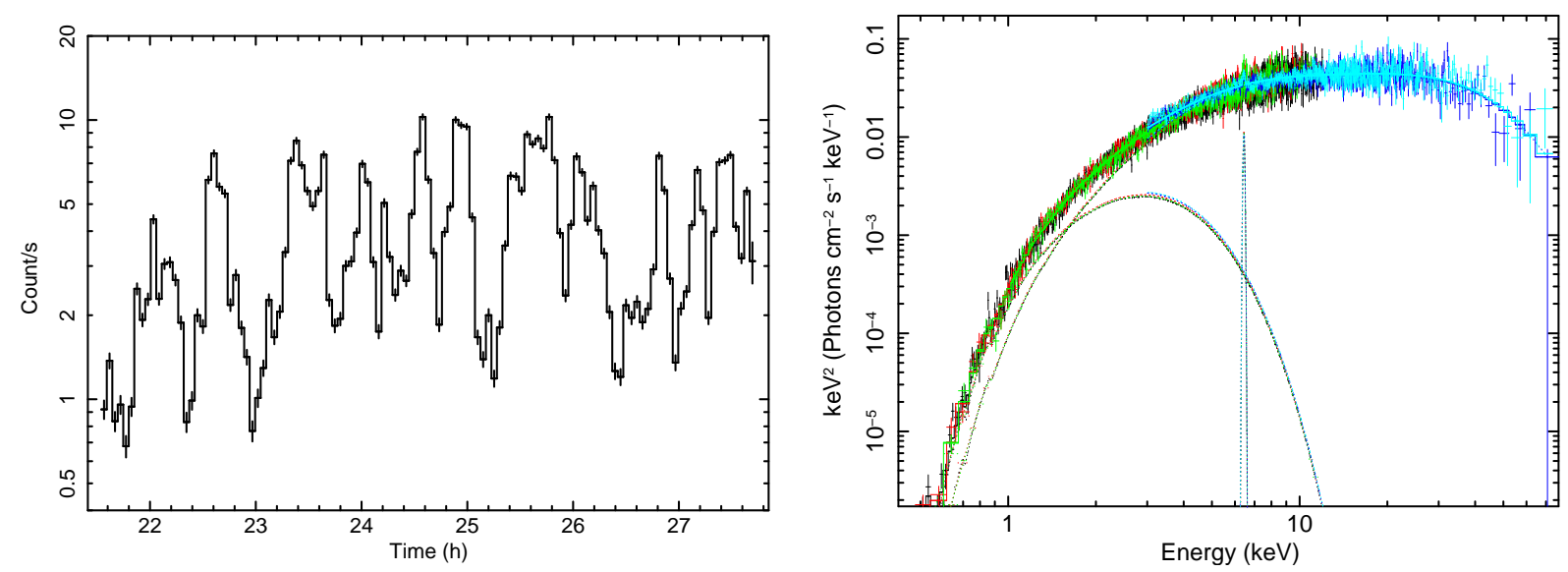

Figure 4: Left panel: EPIC pn light curve of SFXT IGRJ 11215-5952 during the February 2016 periodic outburst (bin time of $187 \mathrm{~s}$, the pulsar spin period). Right panel: Broad-band time-averaged spectrum of the SFXT IGRJ 11215-5952 observed by XMMNewton and NuSTAR in February 2016, fitted with a soft black body $\left(\mathrm{kT}_{b b}=0.7 \mathrm{keV}\right.$ and a radius of few hundred meters at $\left.7 \mathrm{kpc}\right)$, a Comptonized emission (electron temperature $\mathrm{kT}_{e} \sim 9 \mathrm{keV}$ ) and a narrow emission line by neutral iron at $6.4 \mathrm{keV}$ ([प] ]).

An alternative model proposed to explain the SFXT behaviour is the settling accretion regime ([4]], [[ㄷ]) that operates in slow, low luminosity, HMXB pulsars $\left(\mathrm{L}_{X}<4 \times 10^{36} \mathrm{erg} \mathrm{s}^{-1}\right)$. Below this luminosity, the matter captured within the Bondi radius is unable to efficiently cool down (by Compton processes) to penetrate the neutron star magnetosphere by means of Rayleigh-Taylor instabilities. A quasi-spherical shell of hot matter forms above the magnetosphere, and only a reduced accretion rate (with respect to what expected in Bondi direct accretion, that operates at higher $\mathrm{L}_{X}$ ) is permitted, by means of inefficient radiative cooling. The low time-averaged luminosity observed in SFXTs suggests that settling accretion can set-in in these sources. In this scenario, the sporadic bright $\mathrm{X}$-ray flares are produced by the complete collapse of the shell onto the neutron star. The accreted mass needed to fuel the $\mathrm{X}$-ray flare well agrees with the estimated mass of the quasi-spherical shell ([[]6]), without the need for huge wind clumps. Also a correlation between the energy emitted during SFXTs flares and the low time-averaged luminosity has been observed, as predicted by this model ([ए]]).

The trigger for the sporadic instability of the shell has been suggested to reside in the magnetized stellar wind from the supergiant donor: magnetic reconnection can temporarily open the neutron star magnetic field lines, allowing accretion of the accumulated matter on free-fall time scale ([06]).

Cumulative distributions of X-ray flare luminosities observed with INTEGRAL (17-50 keV) support the settling accretion model ([[]]): the exploitation of the whole INTEGRAL archive spanning more than 10 years, allowed us to build meaningful distributions of SFXTs peak flare luminosities. The main results of this systematic study were the full characterization of the SFXT low duty cycles in outburst (0.1-5\%), together with the important findings that the luminosity distributions of the SFXTs flares follow a power-law, contrary to what displayed by SgHMXBs, where the distribution of the $\mathrm{X}$-ray luminosity is log-normal. Power-law-like distributions are reminiscent of self-organized criticality (SOC) systems (see the reviews by [82], [83]]). The prototypical exam- 
ple of a SOC system is a sandpile, where unpredictable sand avalanches occur when an instability threshold is reached by adding sand grains, one by one, to the pile; when a critical slope of the pile is reached, sand avalanches occur and they display many sizes. The resulting size distribution is a power-law ([84]). Also solar flares, among other natural phenomena, are thought to be SOC systems and produce power-law-like size distributions. Since solar flares are produced by magnetic reconnection, SFXTs flares (produced by the collapse of the shell) could be triggered by the interaction of the accreting magnetized stellar wind with the neutron star magnetosphere ([[0]]). Also the temporal properties of flares from SFXTs show cumulative distributions that can be interpreted in the scenario that involves magnetized supergiant winds and their fractal structure [ $[\mathbb{0}]$.

As the magnetic field of the accreting wind material is proposed to play an important role in triggering the flares by magnetic reconnection at the neutron star magnetospheric boundary, also the density and velocity of the clumpy wind have been proposed as a crucial difference with respect to persistent SgHMXBs ([[6]], [[ד]). Systematically faster and less dense winds in massive donor in SFXTs could reduce the X-ray luminosity in order to enable the inefficient radiative cooling regime in the quasi-spherical settling accretion.

It is usually assumed that supergiants in SFXTs and in persistent SgHMXBs are very similar. However, this paradigm needs to be tested observationally, and deserves further multifrequency investigation. Very recently, a comparative study of the wind in the donor stars in IGR J175442619 (the prototype of SFXTs) and Vela X-1 (the prototype of persistent SgHMXBs) revealed that these supergiants have different properties: the wind terminal velocity is observed to be a factor of two higher in this SFXT $\left(1500 \mathrm{~km} \mathrm{~s}^{-1}\right)$ than in Vela X-1 (700 $\mathrm{km} \mathrm{s}^{-1}$, [55]]). A faster wind implies a much lower accretion rate in IGR J17544-2619, with respect to Vela X-1.

These important observations strongly support the idea that the dichotomy of wind-fed HMXBs, between SFXTs and persistent sources, is produced by a dichotomy in the supergiant wind properties [55]. However, further multifrequency observations of members of both classes are needed to provide us with a final answer to the issue, since the X-ray behavior emerges from the specific combination of the properties of both the neutron star (spin and magnetic field) and the supergiant wind (its velocity, density and magnetic field).

\section{Conclusions and future directions}

In this brief review paper I have summarized the most recent achievements in the SFXT field. The two main proposed explanations for the SFXTs phenomenology (gated mechanisms and subsonic settling accretion regime) invoke a dichotomy in the neutron star properties and/or in the wind from the hot massive companion, between SFXTs and persistent SgHMXBs. Neutron star properties in SFXTs remain quite elusive and require new X-ray observations. Given the powerlaw distribution of the SFXTs flare luminosities, the future discovery of more extreme behaviours at X-rays is certain: it is only a matter of (observing) time.

HMXBs are not clean laboratories and the donor and the neutron star are inextricably intertwined: a complex feedback is present between the two protagonists, since also the $\mathrm{X}$-ray emission from the compact object influences the wind structure and its acceleration by means of photoionization ([[]]). For this reason, systematic, simultaneous, orbital-phased resolved, multifrequency observations would be desired to solve the issue of the SFXT variability. In particular, after more 
than a decade from their discovery, a dedicated effort to the investigation of the wind of their supergiant donors is urgently needed.

\section{Acknowledgments}

I would like to thank the organizers for their kind invitation to give this review talk at the XII Multifrequency Behaviour of High Energy Cosmic Sources Workshop, held in Palermo, Italy, in June 2017.

I acknowledge financial contribution from the agreement ASI-INAF INTEGRAL n. 2013025.R.0, from the agreement ASI-INAF NuSTAR I/037/12/0, and from the PRIN-INAF 2014 grant "Towards a unified picture of accretion in High Mass X-Ray Binaries".

\section{References}

[1] R. Walter, A. A. Lutovinov, E. Bozzo and S. S. Tsygankov, High-mass X-ray binaries in the Milky Way. A closer look with INTEGRAL, A\&A Rev. 23 (Aug., 2015) 2], [15505.0365]].

[2] S. Martínez-Núñez, P. Kretschmar, E. Bozzo, L. M. Oskinova, J. Puls, L. Sidoli et al., Towards a unified view of inhomogeneous stellar winds in isolated supergiant stars and supergiant high mass X-ray binaries, ArXiv e-prints (Jan., 2017),

[3] V. Sguera, E. J. Barlow, A. J. Bird, D. J. Clark, A. J. Dean, A. B. Hill et al., INTEGRAL observations of recurrent fast $X$-ray transient sources, $1 \& A 444$ (Dec., 2005) 221-23], [astro-ph/0509018].

[4] I. Negueruela, D. M. Smith, P. Reig, S. Chaty and J. M. Torrejón, Supergiant Fast X-ray Transients: a new class of high mass X-ray binaries unveiled by INTEGRAL, in Proc. of the "The X-ray Universe 2005”, 26-30 September 2005, El Escorial, Madrid, Spain. Ed. by A. Wilson. ESA SP-604, Volume 1, Noordwijk: ESA Pub. Division, ISBN 92-9092-915-4, 2006, 2006.

[5] C. Winkler, T. Courvoisier, G. Di Cocco, N. Gehrels, A. Giménez, S. Grebenev et al., The INTEGRAL mission, $1 \& A 411$ (Nov., 2003) LI-L6.

[6] D. M. Smith, C. B. Markwardt, J. H. Swank and I. Negueruela, Fast X-ray transients towards the Galactic bulge with the Rossi X-ray Timing Explorer, MNRAS 422 (May, 2012) 2661-2674, [1202.6434].

[7] A. Paizis and L. Sidoli, Cumulative luminosity distributions of supergiant fast $X$-ray transients in hard $X$-rays, MNRAS 439 (Apr., 2014) 3439-3452, [1401.686]].

[8] L. Sidoli, A. Paizis and K. Postnov, INTEGRAL study of temporal properties of bright flares in Supergiant Fast X-ray Transients, MNRAS 457 (Apr., 2016) 3693-3701, [1601.07000].

[9] E. Bozzo, P. Romano, L. Ducci, F. Bernardini and M. Falanga, Supergiant fast X-ray transients as an under-luminous class of supergiant X-ray binaries, Advances in Space Research 55 (Feb., 2015) [255-1263, [14411.4470]].

[10] P. Romano, Seven years with the Swift Supergiant Fast X-ray Transients project, Dournal of High Energy Astrophysics 7 (Sept., 2015) 126-136, [1504.07249].

[11] J. J. M. in't Zand, Chandra observation of the fast X-ray transient IGR J17544-2619: evidence for a neutron star?, A\&A 441(Oct., 2005) L1-L4, [arXiv: astro-ph/0508240]. 
[12] R. González-Riestra, T. Oosterbroek, E. Kuulkers, A. Orr and A. N. Parmar, XMM-Newton observations of the INTEGRAL X-ray transient IGR J17544-2619, 4\&A 420(June, 2004) 589-594, [astro-ph/0402293].

[13] S. P. Drave, A. J. Bird, L. Sidoli, V. Sguera, A. Bazzano, A. B. Hill et al., New insights on accretion in supergiant fast X-ray transients from XMM-Newton and INTEGRAL observations of IGR J17544-2619, MNRAS 439 (Apr., 2014) 2175-2185, [10401_.3570].

[14] C. M. Boon, A. J. Bird, A. B. Hill, L. Sidoli, V. Sguera, M. E. Goossens et al., Spectral variation in the supergiant fast X-ray transient SAX J1818.6-1703 observed by XMM-Newton and INTEGRAL, MNRAS 456 (Mar., 2016) 4111-4120, [1601.0159]].

[15] L. Sidoli, A. Tiengo, A. Paizis, V. Sguera, S. Lotti and L. Natalucci, XMM-Newton and NuSTAR Simultaneous X-Ray Observations of IGR J11215-5952, ApJ 838 (Apr., 2017) 133, [1103.05030].

[16] E. Bozzo, F. Bernardini, C. Ferrigno, M. Falanga, P. Romano and L. Oskinova, The accretion environment of Supergiant Fast X-ray Transients probed with XMM-Newton, ArXiv e-prints (Sept., 2017), [1709.00716].

[17] P. Romano, E. Bozzo, V. Mangano, P. Esposito, G. Israel, A. Tiengo et al., Giant outburst from the supergiant fast $X$-ray transient IGR J17544-2619: accretion from a transient disc?, A\&A 576 (Apr., 2015) L4, [1502.0471]].

[18] L. Sidoli, P. Romano, V. Mangano, A. Pellizzoni, J. A. Kennea, G. Cusumano et al., Monitoring Supergiant Fast X-ray Transients with Swift. I. Behavior outside outbursts, ApJ 687 (May, 2008) 1230-1235, [ArXiv:0805.1808].

[19] E. Bozzo, S. Campana, L. Stella, M. Falanga, G. Israel, R. Rampy et al., XMM-Newton and Swift observations of the Supergiant Fast X-ray transient SAXJ1818.6-1703, The Astronomer's Telegram 1493 (Apr., 2008) .

[20] L. Sidoli, A. Paizis and S. Mereghetti, IGR J11215-5952: a hard X-ray transient displaying recurrent outbursts, A\&A 450(Apr., 2006) L9-L12, [astro-ph/0603081].

[21] L. Sidoli, P. Romano, S. Mereghetti, A. Paizis, S. Vercellone, V. Mangano et al., An alternative hypothesis for the outburst mechanism in supergiant fast X-ray transients: the case of IGR J11215-5952, A\&A 476(Dec., 2007) 1307-1315, [arXiv:0710.1175].

[22] P. Romano, L. Sidoli, G. Cusumano, S. Vercellone, V. Mangano and H. A. Krimm, Disentangling the System Geometry of the Supergiant Fast X-Ray Transient IGR J11215-5952 with Swift, ApJ696 (May, 2009) 2068-2074, [0902.1985].

[23] R. Gamen, R. H. Barbà, N. R. Walborn, N. I. Morrell, J. I. Arias, J. Maíz Apellániz et al., The eccentric short-period orbit of the supergiant fast X-ray transient HD 74194 (=LM Vel), A\&A $\mathbf{5 8 3}$ (Nov., 2015) L4, [15]0.06584].

[24] M. E. Goossens, A. J. Bird, S. P. Drave, A. Bazzano, A. B. Hill, V. A. McBride et al., Discovering a 5.72-d period in the supergiant fast $X$-ray transient AX J1845.0-0433, MNRAS 434 (Sept., 2013) 2182-2187, [11307.0709].

[25] L. Sidoli, P. Esposito and L. Ducci, The longest observation of a low-intensity state from a supergiant fast X-ray transient: Suzaku observes IGRJ08408-4503, MNRAS 409(Dec., 2010)611-618, [1007.109]].

[26] S. P. Drave, A. J. Bird, L. J. Townsend, A. B. Hill, V. A. McBride, V. Sguera et al., X-ray pulsations from the region of the supergiant fast X-ray transient IGR J17544-2619, A\&A 539 (Mar., 2012) A21, [120].2284]. 
[27] R. Barba, R. Gamen and N. Morrell, HD 74194, a new binary supergiant fast X-ray transient?, possible optical counterpart of INTEGRAL hard X-ray source IGR J08408-4503, ATel 819 (May, 2006) $1-+$.

[28] J.-C. Leyder, R. Walter, M. Lazos, N. Masetti and N. Produit, Hard X-ray flares in IGR J08408-4503 unveil clumpy stellar winds, A\&A 465 (Apr., 2007) L35-L38, [arXi v: 0712. 1200].

[29] I. Negueruela, D. M. Smith and S. Chaty, HD 306414 and IGR J11215-5952, ATel 470 (Apr., 2005) $1-+$.

[30] J. Lorenzo, I. Negueruela and A. J. Norton, HD 306414, the Optical Counterpart to the Peculiar X-Ray Transient IGR J11215-5952, in High Energy Phenomena in Massive Stars (J. Martí, P. L. Luque-Escamilla and J. A. Combi, eds.), vol. 422 of Astronomical Society of the Pacific Conference Series, p. 259, May, 2010.

[31] J. Lorenzo, I. Negueruela, N. Castro, A. J. Norton, F. Vilardell and A. Herrero, Astrophysical parameters of the peculiar X-ray transient IGR J11215-5952, A\&A 562 (Feb., 2014) A18, [1312.559].

[32] L. Sidoli, A. Paizis and S. Mereghetti, IGR J11215-5952: a hard X-ray transient displaying recurrent outbursts, A\&A 450(Apr., 2006) L9-L12, [astro-ph/0603081].

[33] P. Romano, L. Sidoli, G. Cusumano, S. Vercellone, V. Mangano and H. A. Krimm, Disentangling the System Geometry of the Supergiant Fast X-Ray Transient IGR J11215-5952 with Swift, ApJ696 (May, 2009) 2068-2074, [0902.1985].

[34] J. H. Swank, D. M. Smith and C. B. Markwardt, RXTE PCA Pointed Observations of IGR J11215-5952, ATel 999 (Feb., 2007) 1-+.

[35] M. M. Hanson, P. S. Conti and M. J. Rieke, A Spectral Atlas of Hot, Luminous Stars at 2 Microns, ApJS 107 (Nov., 1996) 281.

[36] A. Coleiro and S. Chaty, Distribution of High-mass X-Ray Binaries in the Milky Way, ApJ764(Feb., 2013) 185, [1212.5460].

[37] M. Fiocchi, V. Sguera, A. Bazzano, L. Bassani, A. J. Bird, L. Natalucci et al., IGR J16328-4726: A New Candidate Supergiant Fast X-ray Transient, ApJ725 (Dec., 2010) L68-L72, [1010.3529].

[38] P. Persi, M. Fiocchi, M. Tapia, M. Roth, A. Bazzano, P. Ubertini et al., On the Near-infrared Identification of the INTEGRAL Source IGR J16328-4726, AJ 150(July, 2015) 21 .

[39] R. H. D. Corbet, S. D. Barthelmy, W. H. Baumgartner, H. A. Krimm, C. B. Markwardt, G. K. Skinner et al., A 10 Day Period in IGR J16328-4726 from Swift/BAT Observations, The Astronomer's Telegram 2588 (Apr., 2010) 1.

[40] M. Fiocchi, A. Bazzano, A. J. Bird, S. P. Drave, L. Natalucci, P. Persi et al., The INTEGRAL Source IGR J16328-4726: A High-mass X-Ray Binary from the BeppoSAX Era, ApJ762 (Jan., 2013) 19, [प1210.3840].

[41] S. Chaty, F. Rahoui, C. Foellmi, J. A. Tomsick, J. Rodriguez and R. Walter, Galactic hard X-ray sources discovered by INTEGRAL brought to light by multi-wavelength observations. I. The nature of the companion star, ArXiv e-prints 0802.1774 (Feb., 2008), [0802.1774].

[42] R. H. D. Corbet, C. Markwardt, L. Barbier, S. Barthelmy, J. Cummings, N. Gehrels et al., Periodicities In X-ray Binaries From Swift/BAT Observations, in Bulletin of the American Astronomical Society, vol. 38 of Bulletin of the American Astronomical Society, p. 335, Sept., 2006. 
[43] L. Sidoli, S. Mereghetti, V. Sguera and F. Pizzolato, The XMM-Newton view of supergiant fast X-ray transients: the case of IGR J16418-4532, MNRAS 420 (Feb., 2012) 554-561, [प110_5218].

[44] R. H. D. Corbet and H. A. Krimm, Superorbital Periodic Modulation in Wind-accretion High-mass $X-R a y$ Binaries from Swift Burst Alert Telescope Observations, 4pJ 778 (Nov., 2013) 45, [1309.4119].

[45] S. P. Drave, A. J. Bird, M. E. Goossens, L. Sidoli, V. Sguera, M. Fiocchi et al., Confirmation of the superorbital modulation of the high mass X-ray binaries 4U 1909+07, IGR J16479-4514 and IGR J16418-4532 with INTEGRAL/IBIS, The Astronomer's Telegram 5131 (June, 2013) 1.

[46] M. J. Coe, J. Fabregat, I. Negueruela, P. Roche and I. A. Steele, Discovery of the optical counterpart to the ASCA transient AX 1845.0-0433, MNRAS 281 (July, 1996) 333-338.

[47] D. J. Clark, V. Sguera, A. J. Bird, V. A. McBride, A. B. Hill, S. Scaringi et al., The orbital period in the supergiant fast X-ray transient IGR J16465-4507, MNRAS 406 (July, 2010) L75-L79, [1005.4763].

[48] V. La Parola, G. Cusumano, P. Romano, A. Segreto, S. Vercellone and G. Chincarini, Detection of an orbital period in the supergiant high-mass X-ray binary IGR J16465-4507 with Swift-BAT, MNRAS 405 (June, 2010) L66-L70, [1005.0684].

[49] R. Walter, J. Zurita Heras, L. Bassani, A. Bazzano, A. Bodaghee, A. Dean et al., XMM-Newton and INTEGRAL observations of new absorbed supergiant high-mass $X$-ray binaries, A\&A 453 (July, 2006) 133-143.

[50] A. Lutovinov, M. Revnivtsev, M. Gilfanov, P. Shtykovskiy, S. Molkov and R. Sunyaev, INTEGRAL insight into the inner parts of the Galaxy. High mass X-ray binaries, $1 \& A 444$ (Dec., 2005) 821-829, astro-ph/0411550].

[51] F. Rahoui, S. Chaty, P.-O. Lagage and E. Pantin, Multi-wavelength observations of Galactic hard $X$-ray sources discovered by INTEGRAL. II. The environment of the companion star, A\&A 484(June, 2008) 801-813, [0802.1770].

[52] E. Nespoli, J. Fabregat and R. E. Mennickent, Unveiling the nature of six HMXBs through IR spectroscopy, A\&A 486 (Aug., 2008) 911-917, [0806.0295].

[53] C. Jain, B. Paul and A. Dutta, Discovery of a short orbital period in the Supergiant Fast X-ray Transient IGR J16479-4514, MNRAS 397 (July, 2009) L11-L15, [0903.5403].

[54] S. P. Drave, D. J. Clark, A. J. Bird, V. A. McBride, A. B. Hill, V. Sguera et al., Discovery of the 51.47-d orbital period in the supergiant fast X-ray transient XTE J1739-302 with INTEGRAL, MNRAS 409 (Dec., 2010) 1220-1226, [11007.3379].

[55] A. Giménez-García, T. Shenar, J. M. Torrejón, L. Oskinova, S. Martínez-Núñez, W.-R. Hamann et al., Measuring the stellar wind parameters in IGR J17544-2619 and Vela X-1 constrains the accretion physics in supergiant fast $X$-ray transient and classical supergiant $X$-ray binaries, $\$$ \& 591 (June, 2016) A26, [1603.00925]].

[56] D. J. Clark, A. B. Hill, A. J. Bird, V. A. McBride, S. Scaringi and A. J. Dean, Discovery of the orbital period in the supergiant fast X-ray transient IGR J17544-2619, MNRAS399(Oct., 2009) L113-L117, [0908.104]].

[57] J. A. Tomsick, S. Chaty, J. Rodriguez, R. Walter and P. Kaaret, Chandra Localizations and Spectra of Integral Sources in the Galactic Plane: The Cycle 9 Sample, ApJ701(Aug., 2009) 811-823,

[0906.2577]. 
[58] A. D’Aì, V. La Parola, G. Cusumano, A. Segreto, P. Romano, S. Vercellone et al., The Swift-BAT survey reveals the orbital period of three high-mass X-ray binaries, A\&A 529 (May, 2011) A30, [1102.4546].

[59] V. Sguera, S. P. Drave, A. J. Bird, A. Bazzano, R. Landi and P. Ubertini, IGR J17354-3255 as a candidate intermediate supergiant fast $X$-ray transient possibly associated with the transient MeV AGL J1734-3310, MNRAS 417 (Oct., 2011) 573-579, [प106.4209].

[60] I. Negueruela and D. M. Smith, Optical counterpart to SAX J1818.6-1703, ATel 831 (June, 2006) 1-+.

[61] J. M. Torrejón, I. Negueruela, D. M. Smith and T. E. Harrison, Near-infrared survey of high mass $X$-ray binary candidates, $A \& A 510(\mathrm{Feb} ., 2010) \mathrm{A61}$, [0910.5603]].

[62] I. Negueruela, J. M. Torrejón and P. Reig, Optical and Infrared characterisation of High Mass X-ray Binaries discovered by INTEGRAL, in Proceedings of the 7th INTEGRAL Workshop, 2008.

[63] J. A. Zurita Heras and S. Chaty, Discovery of an eccentric 30 day period in the supergiant X-ray binary SAX J1818.6-1703 with INTEGRAL, A\&A 493 (Jan., 2009) L1-L4, [081] 294]].

[64] A. J. Bird, A. Bazzano, A. B. Hill, V. A. McBride, V. Sguera, S. E. Shaw et al., Discovery of a 30-d period in the supergiant fast X-ray transient SAX J1818.6-1703, MNRAS 393 (Feb., 2009) L11-L15, [0810.5696].

[65] E. Nespoli, J. Fabregat and R. Mennickent, K-band Spectroscopy of AX J1841.0-0536 and IGR J19140+0951, ATel 983 (Jan., 2007) 1-+.

[66] V. Sguera, S. P. Drave, L. Sidoli, N. Masetti, R. Landi, A. J. Bird et al., X-ray, optical, and infrared investigation of the candidate supergiant fast X-ray transient IGR J18462 - 0223, $A \& A 556$ (Aug.. 2013) A27, [1305.1538].

[67] F. Rahoui and S. Chaty, IGR J18483-0311: a new intermediate supergiant fast X-ray transient, $1 \& \& A$ 492 (Dec., 2008) 163-166, [0809.4415]].

[68] A. M. Levine and R. Corbet, Detection of Additional Periodicities in RXTE ASM Light Curves, The Astronomer's Telegram 940 (Nov., 2006) 1-+.

[69] P. Romano, L. Sidoli, L. Ducci, G. Cusumano, V. La Parola, C. Pagani et al., Swift/XRT monitoring of the supergiant fast X-ray transient IGR J18483-0311 for an entire orbital period, MNRAS 401 (Jan., 2010) 1564-1569, [0909.5109].

[70] V. Sguera, A. B. Hill, A. J. Bird, A. J. Dean, A. Bazzano, P. Ubertini et al., IGR J18483-0311: an accreting X-ray pulsar observed by INTEGRAL, A\&A 467 (May, 2007) 249-257, arXiv:astro-ph/0702477].

[71] L. M. Oskinova, A. Feldmeier and P. Kretschmar, Clumped stellar winds in supergiant high-mass $X$-ray binaries: X-ray variability and photoionization, MNRAS 421(Apr., 2012) 2820-2831, [1201.1915].

[72] S. A. Grebenev and R. A. Sunyaev, The first observation of AX J1749.1-2733 in a bright X-ray state. Another fast transient revealed by INTEGRAL, Astronomy Letters 33 (Mar., 2007) 149-158.

[73] E. Bozzo, M. Falanga and L. Stella, Are There Magnetars in High-Mass X-Ray Binaries? The Case of Supergiant Fast X-Ray Transients, ApJ 683 (Aug., 2008) 1031-1044.

[74] N. Shakura, K. Postnov, A. Kochetkova and L. Hjalmarsdotter, Theory of quasi-spherical accretion in $X$-ray pulsars, MNRAS 420(Feb., 2012) 216-236, [1]10.370]]. 
[75] N. Shakura and K. Postnov, Wind Accretion - Observations Vs Theory, ArXiv e-prints (Feb., 2017), [ए702.03393].

[76] N. Shakura, K. Postnov, L. Sidoli and A. Paizis, Bright flares in supergiant fast X-ray transients, MNRAS 442 (Aug., 2014) 2325-2330, [11405.5707].

[77] K. Postnov, L. Oskinova and J. M. Torrejón, Low-luminosity stellar wind accretion onto neutron stars in HMXBs, ArXiv e-prints (Jan., 2017),

[78] E. Bozzo, L. Oskinova, A. Feldmeier and M. Falanga, Clumpy wind accretion in supergiant neutron star high mass X-ray binaries, 1\&A 589 (May, 2016) A102, [1603.05187].

[79] V. Sguera, L. Ducci, L. Sidoli, A. Bazzano and L. Bassani, XMM-Newton and INTEGRAL study of the SFXT IGR J18483-0311 in quiescence: hint of a cyclotron emission feature?, MNRAS 402 (Feb. 2010) L49-L53, [0912.1730].

[80] V. Bhalerao, P. Romano, J. Tomsick, L. Natalucci, D. M. Smith, E. Bellm et al., NuSTAR detection of a cyclotron line in the supergiant fast X-ray transient IGR J17544-2619, MNRAS 447 (Mar., 2015) [2274-228], [14407.0112]].

[81] E. Bozzo, V. Bhalerao, P. Pradhan, J. Tomsick, P. Romano, C. Ferrigno et al., Multi-wavelength observations of IGR J17544-2619 from quiescence to outburst, A\&A 596 (Nov., 2016) A16, [15610.02648].

[82] M. J. Aschwanden, A Macroscopic Description of Self-Organized Criticality Systems and Astrophysical Applications, ArXiv e-prints (Oct., 2013) , [1310.419]].

[83] M. J. Aschwanden, Order out of Chaos : Self-Organization Processes in Astrophysics, ArXiv e-prints (Aug., 2017), [[1708.03394]].

[84] P. Bak, C. Tang and K. Wiesenfeld, Self-organized criticality - An explanation of $1 / f$ noise, Physical Review Letters 59 (July, 1987) 381-384. 